\title{
Ultrasound in the Diagnosis of Biliary Ascariasis and Its Complications and Correlation of Findings with MRI
}

\author{
Shivani Mahendru ${ }^{1}$, Astha Garg${ }^{2}$, Kritika Agrawal ${ }^{3}$, Yash Patel $^{4}$, Anubha Singh 5 \\ 1, 2, 3, 4,5 Department of Radiodiagnosis, Maharishi Markandeshwar Institute of \\ Medical Sciences and Research, Haryana, India.
}

\section{INTRODUCTION}

Parasitic infection of the biliary tract is a common complication. Ascaris lumbricoides, Clonorchis sinensis, Opisthorchis viverrini, Opisthorchis felineus, and Dicrocoelium dendriticum are closely associate with $C$. sinensis and can also cause serious biliary problems. Fascioliasis, induced by Fasciola hepatica and F. gigantica is a zoonotic helminthiasis that can occur as an acute hepatic or chronic biliary tract infection. ${ }^{1}$

Ascaris lumbricoides is the most common bile duct parasitic infection. ${ }^{1}$ Although Ascaris lumbricoides usually resides in the duodenum and proximal jejunum, they may cross into ampulla of Vater and enter the bile duct, gall bladder or pancreatic duct, causing various complications such as biliary colic, cholelithiasis, cholecystitis, pyogenic cholangitis, liver abscess and pancreatitis. Computed tomography (CT), magnetic resonance imaging (MRI), and ultrasound are useful imaging tools for the diagnosis of these parasites and their complications. Recently, MRCP is being used in diagnosing biliary infection by ascaris.

Life Cycle - Ascariasis is ingested by humans through food, water, or soil contaminated with embryonated eggs. After the ingestion of the hatched eggs, larvae are released in the duodenum. The larvae migrate through the duodenal wall into the bloodstream and then into the pulmonary circulation. They then enter the alveoli, pass up to the bronchi and trachea, and are swallowed to reach the small intestine. The larvae mature within the small intestine to become adults.

Thousands of eggs are laid daily, passed in the feces, contaminating the soil. Ingestion of embryonated eggs completes the life cycle. The eggs are immune to cold weather, chemical water purifiers, disinfectants and can remain viable and contagious for up to 10 years, ${ }^{2}$ making it difficult to eradicate.
Corresponding Author:

Astha Garg,

House No. 1838 Sector-9,

Faridabad, Haryana, India.

E-mail: aasthagarg0808@gmail.com

DOI: $10.14260 /$ jemds $/ 2021 / 68$

How to Cite This Article:

Mahendru S, Garg A, Agrawal K, et al. Ultrasound in the diagnosis of biliary ascariasis and its complications and correlation of findings with MRI.J Evolution Med Dent Sci 2021;10(05):308-311, DOI: 10.14260/jemds/2021/68

Submission 18-09-2020,

Peer Review 13-11-2020,

Acceptance 20-11-2020,

Published 01-02-2021.

Copyright (c) 2021 Shivani Mahendru et al. This is an open access article distributed under Creative Commons Attribution License [Attribution 4.0 International (CC BY 4.0)]

\section{PRESENTATION OF CASE}

A 50-years-old female from Saharanpur, Uttar Pradesh, presented with a history of abdominal pain and cough for 8 days. Her investigations on admission showed an abnormal liver profile: alanine transaminase $44 \mathrm{U} / \mathrm{L}$ (normal 5 - 35), aspartate transaminase $41 \mathrm{U} / \mathrm{L}$ (normal 5 - 35), and alkaline phosphatase $155 \mathrm{U} / \mathrm{L}$ (normal 28 - 112). Ultrasound examination showed a linear echogenic structure in the left hepatic duct with an associated small abscess in the left lobe of the liver. Common bile duct (CBD) was thick-walled with the presence of air loculi and sludge within it. (Figure 1) Magnetic resonance cholangiopancreatography (MRCP) was done thereafter, which revealed a linear hypointense structure in CBD extending into the left hepatic duct (which was likely the worm) with associated cholangitis and cholangitic abscess in the left lobe of the liver with associated pneumobilia. 

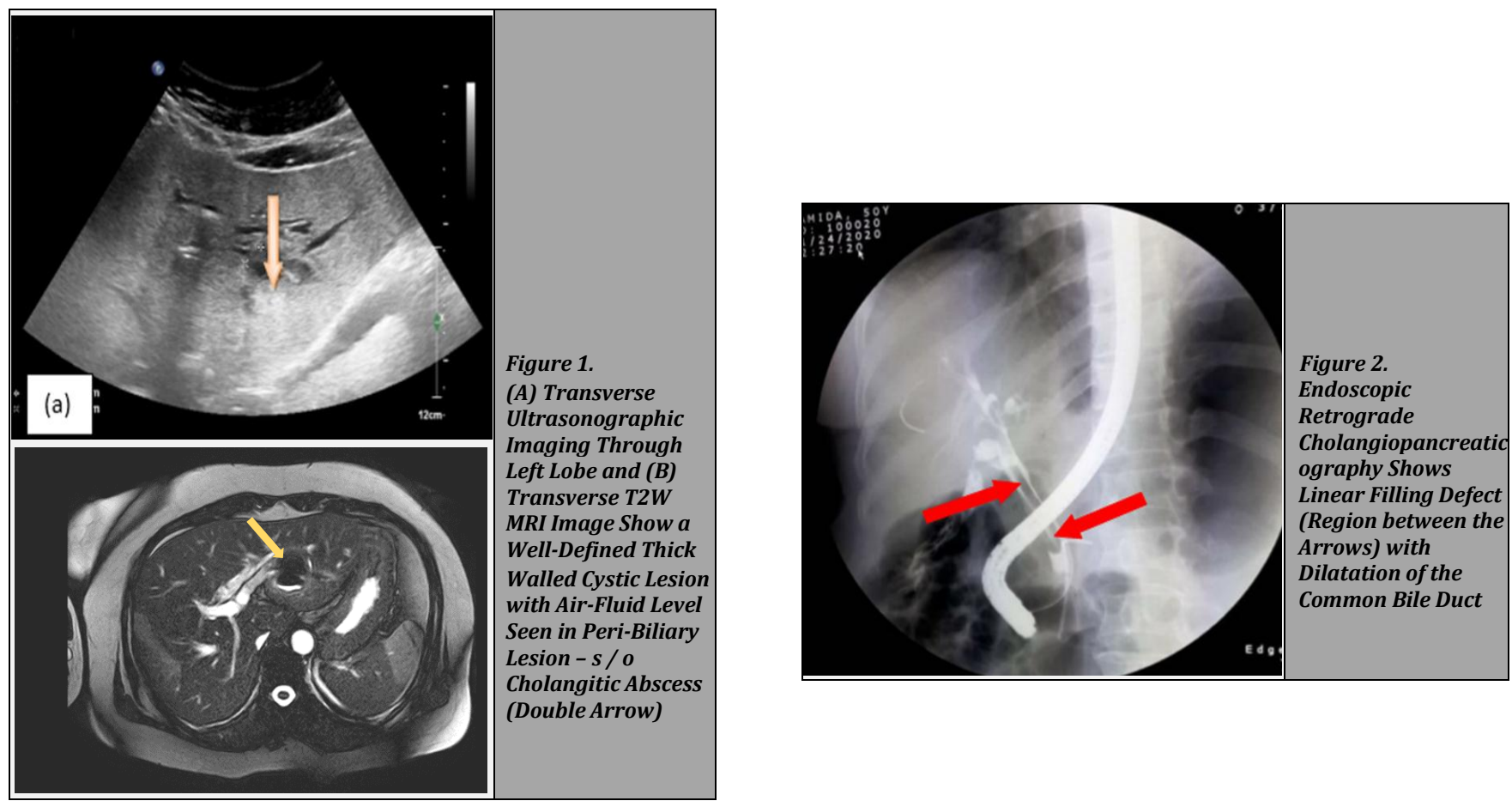

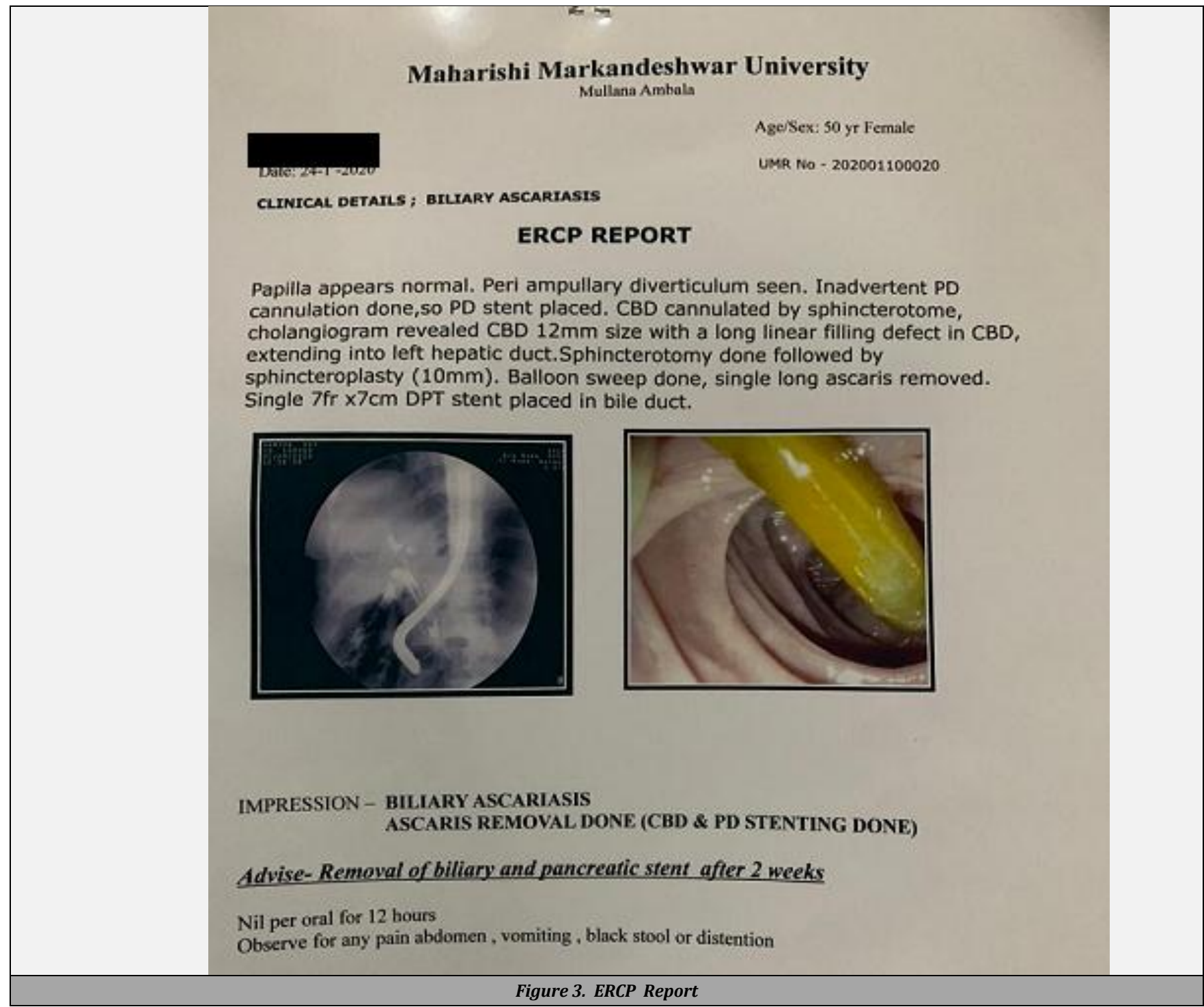




\section{DISCUSSION OF MANAGEMENT}

The patient subsequently underwent endoscopic retrograde cholangiopancreatography (ERCP), which shows a periampullary diverticulum. Inadvertent pancreatic duct (PD) cannulation was done, and a stent was placed in the PD. CBD was cannulated by sphincterotome, and the resultant cholangiogram revealed dilated CBD $(12 \mathrm{~mm})$ with a long linear filling defect in it, extending into the left hepatic duct. Sphincterotomy was done, followed by sphincteroplasty $(10$ $\mathrm{mm}$ ). It was followed by a balloon sweep and the single long ascaris was removed. Single $7 \mathrm{fr}$ X $7 \mathrm{~cm}$ double pigtail (DPT) stent was placed in bile duct. Subsequently, the patient's clinical and biochemical profile improved. She was given a course of oral mebendazole and had no further complications since then.

\section{DISCUSSION}

The most common helminthic infection worldwide is Ascaris lumbricoides. An adult worm is typically $15-50 \mathrm{~cm}$ in length and $3-6 \mathrm{~mm}$ in thickness. During the intestinal phase, the worms may be silent or cause abdominal pain, vomiting, or bowel obstruction. Unusual ascaris worm locations include gall bladder, bile ducts, liver ducts, and pancreatic ducts. When the worm extends across the gall bladder, the ultrasound examination reveals a "septate" gall bladder. A heavy parasitic load causes the worm to migrate to these uncommon positions. $^{3}$ It is more common for a worm to move to the CBD than to the PD, possibly because of the PD's smaller size. Migration of a worm into the biliary tree via the Vater papilla is an unusual complication and contributes to biliary colic, recurrent pyogenic cholangitis, pancreatitis, hepatic abscesses and septicemia. 4 These irritating factors can even lead to cholangiocarcinoma. ${ }^{5}$ Pregnancy predisposes females to hepatobiliary and pancreatic ascariasis.

Previous studies have shown that almost 30 per cent of biliary ascariasis patients have a history of cholecystectomy. ${ }^{6}$ Dilatation of the common bile duct occurs after cholecystectomy, and there is an increase in cholecystokinin, which in turn contributes to relaxation of sphincter of Oddi.

Serum alkaline phosphatase rises early and is the most commonly elevated biochemical parameter to be detected. ${ }^{7} \mathrm{An}$ increase in the eosinophil count and serum immunoglobulin $\mathrm{E}$ (IgE) levels can also be seen in the later part of the migratory process of ascariasis. In the intestinal stage of the disease, these counts fall quickly.

Plain radiography of the abdomen can be normal when the ascarids are few. However, in heavy infestation, the aggregated ascarids may be seen as a group of thick entangled cords contrasted against the bowel gas. On barium inspection, the ascarids appear as elongated, smooth, cylindrical, and sometimes coiled radiolucent filling defects within the bariumfilled intestinal lumen. They are mostly found in the jejunum and ileum. Ascaris may ingest barium in a fasting patient, thereby outlining its alimentary canal as a central thin white line. In heavily infested patients, especially children, clumps of ascarids can be seen filling up the lumen of the small bowel.

Ultrasonography is a handy tool in the diagnosis of biliary ascariasis. Ultrasound of the abdomen is the first investigation to be done in such patients, allowing biliary ascariasis to be diagnosed in approximately $85 \%$ of cases. The patterns on ultrasound include single or multiple long, linear, tubular echogenic strips without acoustic shadowing in the CBD or PD (strip sign); a central, longitudinal, anechoic shadow within the worm (inner tube or double tube sign), spaghetti sign (overlapping longitudinal interfaces in the main bile duct) and a characteristic sinuous movement. Roundworms in CBD sometimes give a pseudotumour appearance.

CT and magnetic resonance imaging, respectively, show the "bull's eye" and "eye-glass" appearances of Ascaris lumbricoides in the CBD.

ERCP is the gold standard investigation for the diagnosis of biliary ascariasis. However, since papillotomy can re-enter the worm into the CBD, it should be reserved for therapeutic rather than diagnostic use. Magnetic resonance (MR) cholangiography has replaced the invasive ERCP as the preferred diagnostic procedure for biliary ascariasis. By using MR cholangiography repeated at 30-sec intervals, live worm movement within the bile duct can be detected. Axial T2 weighted images show a hypointense dot signal in CBD around which the bile signals are hyper intense.

\section{Management}

Conservative Management

Conservative management involves the prohibition of oral feeding, intravenous fluid, analgesics, and antibiotics. Conservative treatment is usually continued for three days. During this time, the migration of the worm should be monitored by serial ultrasonography. Previous studies have shown that $42-90 \%$ of patients could be treated by noninvasive means. ${ }^{7}$ Targeted worm therapy against $A$. lumbricoides should be deferred until the worms move out of the biliary tree.

\section{Endoscopy}

Most writers suggest early intervention with endoscopy as this has resulted in a substantial reduction in morbidity and mortality of this disease. ${ }^{8}$ However, Khuroo et al. ${ }^{9}$ showed that ERCP / endoscopic intervention was required in only $29 \%$ of patients after the failure of conservative management. Grasping the worm by a Dormia basket and extracting it through the papilla leads to rapid relief of symptoms and reduced complication rate. ${ }^{8}$ Endoscopic intervention at an early stage is also an option in cases of pyogenic cholangitis and acute pancreatitis. In cases where the worm completely migrates into the bile ductal system, an ERCP may be indicated. Fragmentation or incomplete extraction of the worm can cause sclerosing strictures of the ducts, leading to failure of endoscopic treatment, where appropriate, sphincterotomy should be avoided, and balloon extraction of worms should be attempted to prevent appropriate future worm re-invasions. The difficulty is encountered when the parasite lies within the intrahepatic ducts. ${ }^{9}$ while dead and calcified worms, which get impacted, are also difficult to extract.

ERCP may be viewed as a therapeutic measure if a patient fails to respond to conservative management or the worm persists (as shown by serial sonograms) or has died inside the pancreato-biliary tree.

\section{Drug Therapy}

Effective drug therapy is available against A. lumbricoides. Direct instillation of the antihelminthic drug into the biliary 
tree is not recommended as it can cause flaccid paralysis and eventual death of the worm within the biliary tree. The macerated worm then elicits a severe inflammatory reaction causing fibrotic strictures. However, these drugs have considerable benefits when the roundworms move out of the biliary tree into the small intestine.

Mebendazole, pyrantel pamoate, piperazine citrate, and levamisole are the different medicines used to treat ascariasis. Of these the most prescribed are mebendazole and pyrantel, citing the least side effects. The effectiveness of treatment can be determined 1 - 2 weeks after drug therapy by a stool test. However, stool samples testing negative does not necessarily mean the eradication of the worm.

\section{Surgical Aspects}

The need for surgical intervention has gradually reduced by introducing ERCP in the management of biliary tract diseases. Rare cases, however, that are not prone to endoscopic extraction require surgery but represent less than $1 \%$ of the scope of treatment. ${ }^{9}$ Other indications include the presence of hepatic-ductal or gallbladder ascarids and acute pancreatitis. Some of the surgical procedures used in the management of intractable biliary ascariasis are choledochotomy, choledochoduodenostomy, and intra-operative biliary duct syringing to raise hydrostatic pressure and promote worm expulsion. Effectively, flush therapy with regular saline injected through a T-tube was used to treat post-operative biliary ascariasis. ${ }^{10}$

\section{CONCLUSIONS}

Ascariasis is the most common parasitic infection. There are ambiguous and non-specific clinical symptoms of ascariasis. Identification of ascaris by various imaging methods helps in the diagnosis and follow up. For intestinal ascariasis, barium studies are the mainstay.

Ultrasound, CT, and other modalities can detect biliary ascariasis, while MRCP is more effective in detecting intraductal ascariasis. They can be seen along the path of CBD as linear hypointensities in the lumen. Endoscopic removal of worms by ERCP is mainly used in an already diagnosed case.

Financial or other competing interests: None.

Disclosure forms provided by the authors are available with the full text of this article at jemds.com.

\section{REFERENCES}

[1] Hashmi MA, De Jevan K. Biliary ascariasis on magnetic resonance cholangiopancreatography. J Global Infect Dis 2009;1(2):144-5.

[2] Das AK. Hepatic and biliary ascariasis. J Global Infect Dis 2014;6(2):65-72.

[3] Sundriyal D, Bansal S, Kumar N, et al. Biliary ascariasis: radiological clue to diagnosis. Oxford Medical Case Reports 2015;2015(3):246-7.

[4] Das CJ, Kumar J, Debnath J, et al. Imaging of ascariasis. Australas Radiol 2007;51(6):500-6.

[5] Rana SS, Bhasin DK, Nanda M, et al. Parasitic infestations of the biliary tract. Curr Gastroenterol Rep 2007;9(2):156-64.

[6] Gonzalez AH, Regalado VC, Van den Ende J. Non-invasive management of ascaris lumbricoides biliary tract migration: a prospective study in 69 patients from Ecuador. Trop Med Int Health 2001;6(2):146-50.

[7] Khuroo MS, Zargar SA, Mahajan R. Hepatobiliary and pancreatic ascariasis in India. Lancet 1990;335(8704):1503-6.

[8] Leung JW, Chung SC. Endoscopic management of biliary ascariasis. Gastrointest Endosc 1988;34(4):318-20.

[9] Khuroo MS, Zargar SA, Yattoo GN, et al. Worm extraction and biliary drainage in hepatobiliary and pancreatic ascariasis. Gastrointest Endosc 1993;39(5):680-5.

[10] Wani NA, Shah OJ, Naqash SH. Post-operative biliary ascariasis: presentation and management--experience. World J Surg 2000;24(9):1143-5. 